\title{
La influencia de Panini en los comparatistas
}

\author{
María Elena Sánchez Arroba \\ Universidad Ricardo Palma \\ El Colegio de México
}

\section{INTRODUCCIÓN}

Un factor que contribuyó al surgimiento de la lingüística comparativa en el siglo XIX fue el interés por el estudio del sánscrito, la antigua lengua literaria de la India, cuya gramática había sido descrita ya desde el siglo IV A.C. por numerosos gramáticos hindúes, entre-los cuales destaca Panini.

En este artículo, me propongo presentar el Astadhyayi, la gramática sánscrita escrita por Panini, y establecer además, la influencia de este autor en la lingüística comparativa de la primera mitad del siglo XIX. Para ello, me baso en la concepción déla estrurcturadedąplabraquesadoptó el comparatista Franz Bopp en sus obras Sobre el sistemacde forjugación del sánscrito en comparación con el griego, el latino, el persa y el alemán (1816) y Gramática Comparada del sánscrito, zendo, armenio, griego, latín, antiguo eslavo, gótico y alemán (1833-1852).

\section{LA LINGÜÍSTICA HINDÚ}

En el siglo XIX, los estudios comparatistas sobre el sánscrito permitieron demostrar el parentesco entre las diversas lenguas y familiarizaron a los europeos con las descripciones gramaticales de los hindúes, que han llegado a influir, incluso en el desarrollo de la moderna linguiística descriptiva. A pesar de que esa tradición gramatical hindú se había desarrollado antes que la 
grecorromana, ambas fueron independientes entre sí; y durante muchos siglos, no se conoció el valor de los análisis lingüísticos hindúes. Bloomfield atribuye esa ignorancia, entre otras razones, al carácter casi ininteligible de las gramáticas hindúes ${ }^{1}$, especialmente el de la obra paniniana que sólo se puede comprender con los comentarios de algún lingüista o del editor.

Hacia fines del siglo XVIII, los sabios occidentales descubrieron las descripciones fonéticas y gramaticales del sánscrito que habían sido elaboradas por representantes de unas doce escuelas distintas de gramática, basadas en diversas doctrinas. Esas escuelas hindúes coincidieron con los gramáticos griegos en la controversia sobre naturaleza y convención en el lenguaje, y en la primacía del lenguaje escrito sobre el oral ${ }^{2}$. Su interés por el estudio de la lengua se debió a razones religiosas, pues en realidad, lo que los gramáticos hindúes intentaban era evitar alteraciones en la pronunciación de los rituales ${ }^{3}$ y de los textos religiosos ${ }^{4}$ que se habían trasmitido oralmente, de generación en generación, desde el periodo védico (1200-1000 A.C.). Este interés, unido a la notable capacidad de análisis de los hindúes, explica la superioridad de la producción lingüística hindú frente a la gramática tradicional de Occidente, especialmente en el estudio de la fonética y de la estructura interna de las palabras. El objetivo metodológico de los gramáticos hindúes fue separar y analizar los distintos niveles de las lenguas. Esa concepción descriptiva se refleja, incluso, en el término hindú con el que nombraron a la gramática: 'vyakarana', que significa "separación", "análisis". Otros rasgos característi$\cos$ de la gramática hindú fueron el énfasis en las relaciones sincrónicas y el deseo de formalizar mediante reglas explícitas, los distintos modos de expresión.

1 BLOOMFIELD, Leonard; sobre Bruno Liebich, Kondornanz Panini-Candra, Breslau, Verlag M. \& H. Marcus, 1928, Language, 5 (1929), p. 268.

2 La historia más completa sobre las gramáticas del sánscrito es la de Yudhisthira Mimamasak, Samakrtavyakarana-sastra ka itihasa (tin bhagom mem purna). (Historia de la gramática del sánscrito), Ajmer Bharatiya Preacyavidya-Pratishana, 1966. Véase Georg Cardona, Panini: a survey of research. The Hague, Mouton, 1976, p. 139.

3 Un error en la pronunciación o en la enunciación de los himnos védicos anulaba por completo el valor sagrado de estos rituales.

4 Las divergencias dialectales habían resaltado aun más las posibles variaciones de estos textos. 
Aunque con frecuencia se ha afirmado que el interés por el estudio del sánscrito en el siglo XIX se debió a la llamada "morfología transparente" de esta lengua, es decir, a una claridad peculiar de su estructura, que habría sido lo que facilitó el análisis; lo cierto es que el estudio del sánscrito resultó sencillo para los occidentales gracias a los aportes de los gramáticos hindúes que ya lo habían analizado.

\section{ASTADHYAYI}

La obra de Panini constituye la culminación de una tradición gramatical que habría empezado varios siglos antes de la fecha en la que supuestamente se redactó el Astadhyayi, o sea, entre el siglo IV y el III A.C. Uno de los problemas más importantes que debe enfrentarse en la investigación de las gramáticas hindúes es el establecimiento de la fecha en que se escribieron las obras; aunque este tema ha perdido ya cierto interés; $y$ en la actualidad, se aceptan fechas probables con las que muchos investigadores están de acuerdo. Para determinar la fecha del Astadhyay, se ha/recurrido a evidencias internas, como lo que Panini dice en sus reglas, y a evidencias externas, como su posible contemporaneidad con otro autor. Entre las evidencias internas, resalta la mención de gramáticos como Apisali e Indra, o la referencia a la escritura griega. Sin embargo, las fechas de estos gramáticos también se conocen sólo aproximadamente; y además, muchos autores dudan sobre la mención de los gramáticos griegos. Una evidencia externa importante es la información sobre

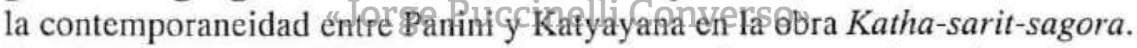
No obstante, estas evidencias resultan poco convincentes y dependen en gran parte de la interpretación. Esa fecha aproximada (siglo IV-siglo III A.C.) se ha establecido tomando como base las características del sánscrito que se describe en el Astadhyayi, un sánscrito muy cercano al de la prosa de los vedas.

La inquietud sobre el sánscrito descrito por Panini se relaciona mucho con el estudio de la literatura hindú. Estudios comparativos realizados por Liebich y Wecker ${ }^{6}$ han mostrado que este sánscrito es anterior al de Brahmanas

5 Sobre ese tema, se puede consultar V.S. Agrawala, "Some chronological considerations about Panini's date", Indian Historical Quaterly, 27 (1951), pp. 264-286.

- LIEBICH, Bruno, "Dic Casushlehre der indischen Grammatiker verglichen mit dem Gebrauch der Casus in Aitareya-Brahmana: ein Beitrag zur Syntax der Sanskrit-Sprache", en Beitrage zur Kunde der Indogermanischen Sprachen, Göttingen, 10 (1886), pp. 205-34, 11, pp. 274 - 
y posterior al de Upanisads; y que además, no corresponde al de ningún texto literario. Esto fue motivo para que Whitney acusara a los gramáticos hindúes de haber creado una lengua artificial, inventando formas que nunca habían existido en el sánscrito.

En realidad, Panini describe el sánscrito coloquial de su época; pero incluye variaciones dialectales de las regiones geográficas cercanas y algunas variantes analizadas por sus predecesores. El sánscrito descrito por Panini corresponde también al sánscrito de los rituales religiosos; aunque no sería acertado afirmar que el Astadhayi haya tenido como fin, preservar una lengua que no era de uso general. Puesto que Panini era originario de Salatura, actualmente Lahur, ciudad ubicada en el noroeste de la India, cerca del río Kabul; la variante descrita en el Astadhyayi correspondería a la de esta región geográfica, que fue un centro de aprendizaje durante varios siglos.

La obra de Panini ha sido calificada por Bloomfield como "uno de los más grandes monumentos de la inteligencia humana"? Muchos lingüistas coinciden en afirmar que esta gramática constituye una descripción lingüística formal verdaderamente cientifica, que destaca por las estrictas definiciones de los términos utilizados, por-sus definiciones abstractas, así como por las formulaciones estrictamente lógicas de los procesos gramaticales.

A pesar de que podría afirmarse que el Astadhyayi constituye uno de los factores decisivos parala ereación de-lálingüistica cemparada, es importante señalar que se trata deJurgstudio piurahientensinceotnico elaborado con fines pedagógicos. La obra contiene aproximadamente $4,000^{8}$ reglas sobre la formación de palabras y oraciones en sánscrito. Este conjunto de fórmulas que incluye recomendaciones para la pronunciación debía ser memorizado y trans-

315. WECKER, Otto; "Der Gebrauch der Kasus in der alteren Upanisad-Literatur verglichen mit der Kassulehre der Indischen Grammatiker", en Beitrage zur Kunde der Indogermanischen Sprache, Göttingen, 30 (1905-1906), pp. 1-61, 177-207. Véase Rossane Rocher, “India"'en Current Trends in Linguistics 13 (1975), p. 6.

7 BLOOMFIELD, Leonard; Lenguaje, trad. Alma Flor Ada de Zubizarreta, Lima, Universidad Nacional Mayor de San Marcos, 1964, p. 224.

8 La cantidad exacta de reglas contenidas en el Astadhyayi ha sido tema de discusión en diversas investigaciones. Consúltese al respecto K. Madhava Krishna Sarma, Panini, Katyayana and Patanjali, Delhi, Sri-Lala Bahadur Shastri Rashtriya Sanskrit Vidyapeeth, 1968. 
mitido mediante la enseñanza oral, por maestros que añadían sus propios comentarios.

En los últimos años, ha surgido una controversia sobre la finalidad de las gramáticas hindúes, en especial, sobre la del Astadhyayi de Panini. Se ha sostenido que los hindúes deseaban ser dignos de mérito ('dharmas') mediante el conocimiento y el uso correcto del lenguaje; pero también se les ha atribuido fines mágico-religiosos que algunos autores han rechazado. Lo cierto es que estas gramáticas no poseen ningún carácter didáctico para los occidentales; por lo que actualmente, ni siquiera los admiradores de Panini utilizan el Astadhyayi para introducir a sus alumnos en el estudio del sánscrito.

Si bien la motivación de Panini habrían sido los cambios sufridos por el sánscrito en el transcurso del tiempo, difícilmente podría afirmarse que el Astadhyayi haya tenido una orientación propiamente histórica. Panini no se propuso explicar la evolución de lâ lengua, sino ofrecer un análisis formal de los elementos constituyentes del sánscrito. La obra de panini es esencialmente descriptiva y sincrónica; y sólo se centra en una lengua. De manera que no tiene casi nada en común con las comparaciones de lenguas y los árboles genealógicos de la lingüística comparativa. Los comparatistas desarrollaron sus propios métodos; pero antes de hacerlo, asimilaron el análisis gramatical del sánscrito elaborado por los hindúes.-

Panini tampoco sibliotecap proper una explicasión filosófica del lenguaje, por lo que Benvenifié sostiene que ePAstadhyayi constituye el "antepasado" de las actuales investigaciones lingüísticas, en especial del estructuralismo que evita reflexiones "mentalistas" para atenerse al registro y el análisis formal de la lengua ${ }^{9}$. El carácter formal de esta gramática se manifiesta también en fórmulas similares a las de las matemáticas, las cuales causan mucha dificultad para comprender la obra paniniana.

Panini utilizó dos clases de símbolos; constantes y variables limitadas, cuya interpretación requiere el conocimiento de las reglas metalingüísticas que él aplica. Como su gramática tuvo carácter oral, Panini utilizó sonidos o patrones de sonidos con el valor de símbolos. Dividió todos los sonidos del

9 BENVENISTE, Emile; Problemas de lingüistica general, trad. Juan Almela, México, Siglo XXI, 1974, p. 33. 
sánscrito en catorce subgrupos con los que creó un sistema de símbolos que le permitió expresar con brevedad, ideas sumamente complicadas. Cada subgrupo de sonidos se caracteriza por un sonido específico que posee el valor de signo, e interviene en la formación de los símbolos llamados "IT".

Estos marcadores "IT" se unen a las formas que poseen los rasgos simbolizados de carácter no fonético y se utilizan para diversos fines, como indicar la pertenencia de los ítems marcados a una clase determinada, señalar la aplicación de una determinada operación, distinguir unidades homófonas, o formar abreviaciones que se refieren a los miembros de grupos ordenados. No constituyen parte del ítem lingüístico, pues sólo aparecen acompañándolo en las reglas gramaticales. Además, después de aplicarse la operación indicada por la regla, estos símbolos deben eliminarse.

Se incluyen, así, muchos elementos metalingüísticos que deberán eliminarse en las formas derivadas durante la etapa final; y de este modo, adquieren carácter explícito las metarreglas, cuya función es describir cómo deben manipularse y aplicarse las regla ${ }^{10}$. Diversos lingüístas han intentado interpretar estas reglas, especialmente a partir del siglo XX, al proponerse demostrar el carácter descriptivo y científico del Astadhyayi.

La obra consta de ocho ('asta') capítulos ('adhyaya'), cada uno de los cuales está dividido en cuatro partes. Además de, las 4,000 reglas llamadas 'sutras', presenta una lista de las racees nominales llamada 'gana-patha' y una lista de las raíces verbales, conocida como dhatu-patha'. Las 4,000 'sutras' están precedidas por el catálogo de sonidos que se subdivide en 14 grupos y se conoce con diversos nombres: 'pratyahara-sutras', 'siva-sutras' y 'mahesvara-sutras'. Se ha discutido mucho si estas tres listas fueron compiladas por una sola persona, que habría sido Panini, o si constituyen en realidad, textos distintos con autores diferentes.

El Astadhyayi presupone un largo período de estudios fonológicos y gramaticales. Es por ello que también se ha discutido sobre el grado en que este autor habría estado influenciado por sus predecesores. En el Astadhyayi,

10 Una explicación de este sistema de símbolos utilizado por Panini puede consultarse en S. $\mathrm{S}$. Barlingay, A modern introduction to Indian Logic. N. Delhi, National Publishing House, 1965. 
Panini menciona las observaciones de algunos maestros y hace referencia a Apisali, Kasyapa, Gargya, Galava, Cakravarmana, Bharadvaja, Sakatayana, Sakalya, Senaka, y Sphotayana. Según los comentaristas, esas referencias tenían como fin indicar diferencias de uso, es decir, aspectos gramaticales de carácter opcional ${ }^{11}$. Muchos investigadores sostienen que con estos nombres, Panini habría hecho mención a las distintas escuelas de gramática. Lo importante es que Panini incluyó en su trabajo usos particulares ya descritos por sus predecesores. En algunos casos, utilizó, incluso, términos que él no había introducido en sus propias reglas. Sin embargo, no se ha podido encontrar alguna formulación de reglas gramaticales previa a Panini que pudiera haber sido copiada por este autor.

Aunque algunos científicos han sostenido que las 'siva-sutras' no fueron obra de Panini, sino de un predecesor, G. Cardona afirma que la investigación sobre la relación entre esos sonidos y las reglas del Astadhyayi ha mostrado que entre cada una de ellas, existe una estrecha relación ${ }^{12}$. La polémica ha sido mayor para el caso de las 'dhatu patha' En esta lista, las raíces aparecen acompañadas de entradas con su significado, pero los comentaristas no están de acuerdo respecto al carácter original de esas entradas. El estudio de las llamadas "falsas raíces" del 'dhatu-patha' ha sido objeto de atención, porque se sabe que muchas de las raíces incluidas no aparecían en el texto que recibió Katyayana.

Pawte ${ }^{13}$ ha atribuido el 'dhatu-patha' a la escuela etimológica, basándose en que muchas de las reglas incluidas resultan innecesarias para la aplicación de otra, y algunas de ellas contradicen incluso a las otras reglas. También incluye como argumento el orden peculiar en el que se organizan las raíces en el 'dhatu-patha'. Cardona, sin embargo, se inclina a aceptar esta lista como paniniana, refiriéndose a las dificultades para descubrir las reglas innecesarias, y a lo absurdo que resulta asignar las raíces a otro autor, sólo porque no se conocen las razones por las cuales las raíces aparecen en ese

11 Panini utilizó tres palabras para expresar los distintos grados de opcionalidad 'va', vibhasa', 'anyatarasyam'. Sobre el tema, véase Paul Kiparsky, Panini as a Variationist, Cambridge, Massachussets Institute of Technology, 1979.

12 CARDONA, Geog; ob. cit., p. 30.

13 PAWTE, I. S.; The structure of the Astadhyayi, Ilubli, 1935. 
orden peculiar ${ }^{14}$. Cardona sostiene, pues, que los argumentos en los que se basa Pawte para atribuir el 'dhatu-patha' a los predecesores de Panini no son convincentes. Los estudios sobre esta lista han consistido en análisis comparativos, especialmente sobre las dos listas que incluye: la lista exhaustiva ('parganana') y la lista tipo ('akrtigana').

La primera traducción del Astadhyayi a una lengua europea, el alemán, se publicó en 1840. En esta edición de dos volúmenes, Otto Böhtlingk añadió varios índices y algunos comentarios sobre las 'sutras' ${ }^{15}$. Posteriormente, en 1887, apareció una nueva edición de esta traducción alemana ${ }^{16}$. En 1882 , se publicó una traducción al inglés preparada por W. Goonatileke ${ }^{17}$; y en 1891, la versión inglesa de Srisa Chandra Vasu ${ }^{18}$. Entre 1848 y 1954, apareció en tres fascículos una versión francesa preparada por Louis Renou ${ }^{19}$; y en 1966, se publicó una nueva edición de esta versión en la que se incluye el texto en sánscrito de las sutras ${ }^{20}$. La versión inglesa en la que aquí me baso fue publicada en 1987 por Sumitra M. Katre ${ }^{21}$, quien se propuso cubrir la necesidad de una edición que presentase el texto de las sutras en alfabeto latino.

14 Cardona, Op. cit., pp. 161-164.

1s Panini's acht Bücher grammatischer Regelen, ed. y trad. Otto Böhtlingk; Bonn, Konig, 1839-1840.

16 Panini's Grammatik, herausgegeben. ubserselzl, enlauter und mit verschiedenen Indices versehen, ed. y trad. Otto Buhtlingk, Lipzig, Hacssel, 9887. Louis Renou ha señalado

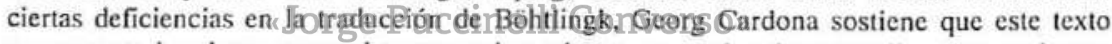
no presenta los datos necesarios para saber cómo y en qué orden se aplican las reglas ya que en algunas reglas, no se incluyen referencias a las otras reglas con las cuales se relacionan, lo cual oscurece el orden de su aplicación.

17 Panini's eight books of grammatical sutras, ed. y trad. W. Goonatileke, Bombay, Education Society Press, 1882.

18 The Astadlhyayi of Panini, ed. y trad. Srisa Chandra Vasu, Alahabad, The Panini Office, 1891.

19 La grammaire de Panini traduite du sanskrit avec des extraits des commentaires indigenes, ed. y trad. Louis Renou, 1948-1954, Georg Cardona opina que esta versión presenta los mismos problemas que la de Böhtlingk, aunque en menor grado. Una crítica sobre esta versión puede consultarse en Paul Thieme, "Panini and the Paniniyas", Journal of the American Oriental Society, 76 (1956), pp. 1-23.

20 La grammaire de Panini traduite du Sanskrit avec des extraits des commentaires indigenes, ed. y trad. Louis Renou, Paris, Ecolc Française d'extreme Orient, 1966.

21 Astadhyayi of Panini, ed. y trad. Sumitra Mangesh Katre, Austin, The University of Texas Press, 1987. 
En efecto, la edición de este autor resulta asequible para quienes no conocemos la escritura silábica del sánscrito ('devanagri').

\section{LAS 'SUTRAS' O REGLAS DEL ASTADHYAYI}

Una de las dificultades para la comprensión de la obra paniniana radica en el estilo de las 'sutras' o reglas, oraciones breves o aforismos sin verbos, que presentan las definiciones o los procesos que ocurren en la formación de palabras. En general, la composición gramatical hindú tuvo como principio básico la búsqueda de economía. Este principio se refleja claramente en el estilo de las 'sutras' paninianas tan difíciles de memorizar; lo cual se opone a los supuestos fines pedagógicos del Astadhyayi. Bahuiikar postula que la dificultad se debería al hecho de que estas 'sutras' habrían sido compuestas en la época inicial de la estructura hindú22.

Las reglas están ordenadas en secuencias; de tal manera que el sentido de cualquiera de ellas es definido o limitado por las anteriores, y constituye además, la base para la aplicación de las siguientes reglas. Deben aplicarse, pues, en un orden fijo. Un principio importante es que una vez dada una regla, ésta sólo puede requerir para su interpretación, información que haya aparecido en alguna de las reglas anteriores. Este ordenamiento es similar al que actualmente propone la gramática generativa en la descripción de los diversos componentes de las lenguas, lo que ha motivado ef renacimiento del interés por la obra de Panini." Jorge Puccinelli. Converso"

Las reglas se formulaban de modo que automáticamente se obtuvieran las formas correctas, y éstas se adecuaran a lo que se definía. Los dos tipos principales de reglas son las operacionales ('vidhisutra'), relacionadas con las operaciones que deben aplicarse a los elementos; $y$ las auxiliares, que facilitan la interpretación y la aplicación de las reglas. Entre estas últimas, aparece la metarregla $1.4 .2 .^{23}$, con la cual se establece que en caso de contradicción entre

22 BAHULIKAR, Saudamani; "Concerning the structure of Panini's Astadhyayi", Indian Linguistics, 34 (1973), p. 96.

23 "1.4.2. Vipratisdhé 'páram karyam. When there is incompatibility (vipratiscdhé) (between two rules which can equally apply in a given domain) what is provided by the subsequent rule (páram) should operate (karyám)" (Panini, Astadhyayi of Panini, ed. y trad. Sumitra Mangesh Katre, Austin, The University of Texas Press, 1987). 
dos reglas, debe aplicarse la última. Esta metarregla se aplica, por ejemplo, en la aparente contradicción entre las reglas 7.3.102, y 7.3.103., cuya comprensión requiere el conocimiento de la regla 7.3.101.

La regla 7.3.101. ' $a T$-ah dirghá-h yaÑ- $i$ ' establece que la vocal breve y final "a" de un tema verbal se alarga cuando le sigue cualquiera de los sonidos nasales, la semivocales, o los aspirados jh, bh. Ejemplos: 'pacami' > 'pacaami'. En la regla 7.3.102. 'suP-i ca'; se señala que la vocal breve y final "a" de un tema nominal se alarga cuando le sigue una terminación de caso que empieza por los sonidos indicados en la regla anterior. Ejemplo 'vrksaya'> 'vrksaaya'. Según la regla 7.3.103. 'bahu-vacan-é jhaL-i eT', la vocal breve y final "a" de un tema nominal se sustituye por -e en plural, cuando le sigue un caso que empieza por una consonante no nasal. En el caso de 'devábhyah', si se aplicara la regla anterior (7.3.102. *devaabhyah'), ya no podría aplicar la 7.3.103. porque se habría perdido la vocal breve "a". Siguiendo la metarregla 1.4.2., se obtiene 'devbhas'> 'devébhyas'. Estos casos muestran cómo para la aplicación de las reglas, setenía en cuenta el contexto, el cual se expresaba metalingüísticamente con los marcadores simbólicos.

\section{PANINI Y LOS COMPARATISTAS}

Los europeos que aprendieron el sánscrito utilizaron gramáticas elaboradas con el sistema de Panini, como en el caso de William Jones. El análisis paniniano de esta léngua fue Gal base den los anàlisis de otras lenguas indoeuropeas y de la reconstrucción del hipotético protoindoeuropeo. La influencia del Astadhyayi en la lingüística del siglo XIX parece haberse centrado en la rigurosa descripción gramatical y en la precisión fonética. W. Allen ha resaltado, por ejemplo, el valor de los conocimientos fonéticos que ofrece Panini en el Astadhyayi $i^{24}$.

Sin embargo, durante la primera mitad del siglo XIX, los comparatistas no ofrecieron serios conocimientos fonéticos, pues hablaban de cambio de letras y no, de cambio de sonidos. La labor comparativa de aquella época se centró en la morfología flexional, tal como aparece en la obra de Bopp.

24 ALLEN, William S.; Phonetics in Ancient India, London, Oxford University Press, 1965 , p. 3. 
Precisamente la gramática de Panini destaca por su atención primordial a la estructura de las palabras.

Las descripciones hindúes también coinciden con las de los comparatistas en haberse basado fundamentalmente en la observación y no sólo, en puras reflexiones teóricas. En general, es fácil descubrir que muchos aspectos de la linguística del siglo XIX proceden directamente de la práctica o de la teoría de los gramáticos hindúes.

La influencia de Panini en los lingüistas comparatistas del siglo XIX se revela claramente mediante el análisis de las nociones de "raíz", "flexión" y "derivación", presentes en el Astadhyayi y en la obra clásica de la gramática comparativa, en la que Bopp se propuso como objetivo la descripción comparada del sánscrito para llegar a conclusiones de tipo histórico.

\section{LA ESTRUCTURA DE LA PALABRA EN PANINI}

Los aportes más importantes de la obra paniniana se encuentran en la morfología. Panini presenta un análisis de la palabra basado en tres unidades fundamentales: raíces nominales o 'pratipadija'25 ('purusa'), raíces verbales o 'dhatu'26 ('bhu', "ser", "convertirse en") y afijos o 'pratyaya'27 ('-ya', desiderativo verbal). Esta identificación de raíces-y de sufijos fue el punto de partida para una investigación propiamente linguistica sobre formación de palabras, en el marco "de la gramática comparativă, y sirvió de inspiración directa para llegar al concepto de morfema.

25 "1.2.45 arthavåd á-dhatur á-pratyayah pratipadikam. (The t.t.) pratipadikáa 'nominalstem' denotes a meaningful (artavát) expression other than a dhatu 'verbal stem' (1.3.1.; 3.1.32) or a pratyayá 'affix' (3.1.1. $-5.4 .166)$.

1.2.46 krt thaddithá-smasas ca. [(The) pratipadiká 45] also (ca) denotes (derived nominal stems ending in $1.1 .72 \mathrm{krt}$ primary affixes $(3.1 .93 \mathrm{ff})$ or those forming samasas 'compounds' (2.1.3. ff)2" (ibid, pp. 42-43).

2t "1.3.1 bhúvadayo dhátavah. (The t.t.) dhatu-verbal base or stem root denotes the class of expressions whose first member is bhú-'become' (1.1).

3.1.32. sáñ-adi-antah-dhatav-ah-. [(The t,t,] dhátu denotes all itemsl, which [end in 1.1.72 the affixes 1] beginning with saN (5-30)" (ibid., pp. 51-88).

27 “pratyayáh (The t.t.) pratyayá 'affix"” (ibid., p. 180). 
En la subclasificación de cada una de estas unidades, puede advertirse también la distinción entre raíz y tema, además de un acercamiento a la distinción entre derivación y flexión, tema actualmente en discusión. Panini clasifica las raíces nominales y las verbales en primitivas y derivadas, según reciban determinados afijos o no. La adición de estos afijos se efectúa de acuerdo con el significado que se desea denotar, según el contexto, o según ambos criterios. Las clases de afijos que presenta Panini pueden analizarse en los capítulos 3, 4 y 5 del Astadhyayi. En esa clasificación general, se observa que el criterio básico es la naturaleza de la raíz a la cual se añaden los afijos, lo que refuerza la separación entre raíz y afijos, así como la distinción entre nombres y verbos; pero no permite vislumbrar una posible distinción entre flexión y derivación.

No obstante, la formación de raíces derivadas mediante determinados afijos que en algunos casos, cambian-la categoría de la raíz revela un acercamiento a la noción de morfemas no flexivos. Estos afijos añaden un significado diferente y crean una construcción a la que se pueden unir las terminaciones de caso. Esto ocurre, por ejemplo, con los sufijos verbales causativos y desiderativos, así como con el sufijo nominal agentivo, lo que puede observarse en los ejemplos del cuadro 1 .

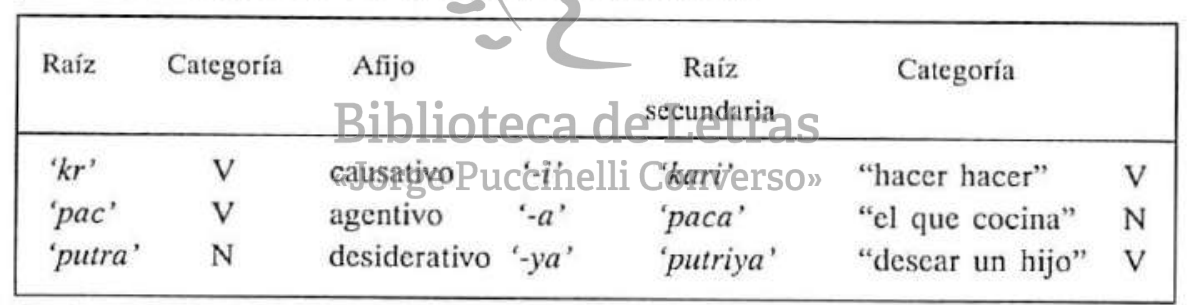

Cuadro 1: Derivación mediante sufijos verbales y nominales.

Puede advertirse que estos afijos equivalen a morfemas derivativos, lo que revelaría una probable analogía entre la dicotomía flexión/derivación y la clasificación entre estos afijos y los afijos 'vibhakti' que presenta Panini.

Estos afijos 'vibhakti' que no forman raíces derivadas parecen coincidir con el concepto de morfemas flexivos. Los afijos 'vibhakti' ${ }^{28}$ son las termi-

28 "1.4.101. TiNas tríni tríni prathamá-madhamá=uttamáh. [The t.tt. 1] prathamá 'third', madhyamá-'second' and uttamá 'first' respectively (1.3.10) denote the three (and = three triplets of tiñ [parsmaipadá and atmanepadá 99-100]. 
naciones propias de los verbos o de los nombres. Los afijos que se añaden a las raíces nominales indican caso (sUp) y los que se añaden a las raíces verbales expresan las diferencias de persona, número y tiempo (tiÑ). En ambos casos, se añaden significados gramaticales y no se crean palabras de distinta categoría sintáctica, o con un contenido semántico distinto del de la raíz. Estos afijos pueden añadirse, tanto a las raíces primitivas como a las derivadas. Cuando se añaden a las raíces derivadas, se percibe claramente la estructura: [raíz + afijo temático + afijo 'vibhakti'], que revela el rasgo periférico característico de los morfemas flexivos.

Esto podría confirmar el carácter flexivo de los afijos 'vibhakti', ya que como sabemos, tres de los argumentos más importantes en favor de la distinción flexión/derivación nos dicen: (1) "Las RF (morfemas flexivos) nunca cambian la categoría sintáctica de la palabra, las R.D. (morfemas derivativos) sí pueden cambiarla"29. (2) "La flexión es siempre periférica con respecto a la derivación" ${ }^{30}$. (3) "Las R.D. (morfemas derivativos) cambian el significado conceptual de la base y las R.F. (morfemas flexivos), únicamente su significado gramatical ${ }^{31}$.

Aunque el límite entre đerivación y flexión es un tema muy discutible y probablemente, un análisis exhaustivo de las clases de afijos en la obra de Panini muestre casos que contradigan esta hipótesis; lo cierto es que el gramático hindú reconoció muy bien las unidades significativas mínimas y pudo distinguir entre aquellas formas lingưisticas bâsicas que no admiten subdivisión, o sea, las raíces, 'y aquentles formas que-se-forman al combinarse con determinados afijos de carácter no flexivo. El concepto de raíz derivada (nominal o verbal) en la obra de Panini puede considerarse análogo al que actualmente se conoce como "tema" o "base" en lingüística moderna. Si bien la terminología varía mucho en esta ciencia según las distintas teorías, muchos

1.4.102 táni-ekavacn'a-dvivacn'a-bahuvacanáni eksah. Those (t'ani-[three and three triplets of tiñ 101] taken one by one (ekavacná 'singular', dvivacaná 'dual' and bahuvacaná 'plural' to denote them respectively (1.31.10)" (ibid., p. 102).

29 SCALISE, Sergio, Morfología Generativa, trad. José Pazó, Madrid, Alianza Universidad, 1987 , p. 119.

30 Ibid., p. 120.

31 Ibid., p. 130. 
lingüistas coinciden en la noción de tema como aquella parte de la palabra a la cual se añaden los morfemas flexivos ${ }^{32}$.

En las raíces verbales, Panini distinguió una noción de tema semejante a la de las gramáticas clásicas, al reconocer una unidad constituida por la raíz básica más un afijo marcador de clase, del cual dependía la adición de los afijos indicadores de tiempo, número y persona. La descomposición de los verbos implicaba un análisis más complejo debido al carácter flexivo del sánscrito.

Cabe recordar que Panini no define claramente estas unidades, puesto que su fin era indicar las reglas para formar palabras a partir de una sucesiva adición de ciertas unidades, o mediante procesos de sustitución. Por ello, no es sencillo establecer una analogía exacta entre las unidades morfológicas de la lingüística y las unidades presentes en el Astadhyayi.

Como el sistema derivativo propuesto por Panini explica, mediante la adición de afijos, tanto la derivación de palabras como la de oraciones: se ha afirmado con frecuencia que Panini sólo analizó las palabras y no distinguió entre morfología y sintaxis. Al respecto, es preciso indicar que los afijos 'karaka' tuvieron como funciôn establecer la relación gramatical entre las palabras. Por otra parte, la distinción entre derivación y flexión constituye uno de los argumentos a les cuales recurre la lingüística general para establecer la relación entre moffología y sintaxis. Si bien en la obra de Panini, la

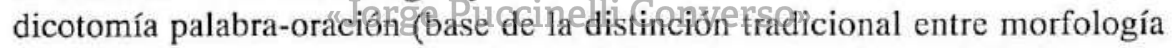
y sintaxis) no aparece de modo explícito; sí se establece el límite entre palabras y oraciones mediante recursos como la pausa final, los sandhis externos, el tono y la cantidad vocálica de los sonidos que aparecen al final de una oración, y otros.

32 En morfología generativa, ha surgido una discusión sobre la unidad que se toma como base para la formación de palabras. Algunos parten de la palabra, como en el caso de Jackendoff (1975), Booij (1977), Scalise (1980), Zwanenburg (1980) y Muysken (1980). En favor del "tema" como base para la derivación de palabras nuevas, se encuentran Siegel (1974), Allen (1978), Hoekstra (1979), Lieber (1980), Williams (1981), Kiparsky (1982) y Selkirk (1982). Estos últimos incluyen temas en el lexicón, además de palabras. Sin embargo, el reconocimiento del tema en el marco de la morfología generativa no supone necesariamente una distinción entre "raíz" y "tema". 
Entre otras precisiones morfológicas de la obra paniniana que coinciden con los análisis de la lingüística descriptiva moderna, cabe destacar también la representación del morfema cero, pues Panini distinguió en la derivación de las 'sutras', formas regulares en los niveles más abstractos de representación que carecían de representación fonológica en la superficie después de aplicarse reglas que la impedían, como en el caso que aparece en el cuadro 2.

\begin{tabular}{|cccc|}
\hline Regla aplicada & Raíz & Suf. temático & Suf. Flexivo \\
\hline R.3.3.62. & 'bhaj' & $\mathrm{V}$ & '-am' \\
R.6.1.67. & 'bhaj' & $\mathrm{O}$ & '-am' \\
\hline
\end{tabular}

Además, analizó morfofonológicamente variantes alomórficas de las raíces y de los afijos, ya que al observar variaciones formales entre elementos funcionalmente equivalentes, Panini estableció formas básicas originales ('Sthanim') que se convertían en los morfos resultantes por medio de reglas de sustitución, semejantes a las reglas de cambio morfofonológico que propone la lingüística moderna. Distinguió, también, los cambios fonéticos y fonológicos que se presentan al unirse palabras o al unirse las unidades morfológicas dentro de una palabra, lo que corresponde a los conceptos de "sandhi externo" y "sandhi internos" de la fonología actual. Todos estos aportes de Panini influyeron, incluso, en Leonard Bloomfield, cuyo método morfofonémico de descripción se asemeja al que se presenta en el Astadhyayi. "Jorge Puccinelli Converso"

Aunque los lingüistas del siglo XIX supieron valorar algunos de los conceptos y métodos gramaticales hindúes, éstos se limitaron a aprovechar las distinciones entre raíz y tema, y en algunos casos, a precisar el concepto de flexión.

\section{LA MORFOLOGÍA DE BOPP EN RELACIÓN CON LA OBRA DE PANINI}

Bopp coincidió con Panini en la distinción entre raíz y tema, distinción que no se observa en las gramáticas grecolatinas, para las cuales, la clasificación de las palabras era un aspecto fundamental más importante que la estructura. En estas gramáticas, las partes de la oración eran subclasificadas de acuerdo con una o más características variables, los denominados 
'accidentia' que incluían variaciones léxicas y flexivas ${ }^{33}$. La forma básica a partir de la cual se derivaban las palabras no era una raíz, sino una forma seleccionada como tal por razones semánticas. En el caso del verbo, por ejemplo, la forma básica era la primera persona singular del presente indicativo activo, que se asociaba en clases con otras palabras individuales formando modelos a partir de los cuales, se podían predecir por analogía formas nuevas. Un modelo similar se aplicó para los nombres (sustantivos y adjetivos), cuya forma básica fue el nominativo singular. Con este modelo tradicional conocido como "Palabra y Paradigma", no podía apreciarse claramente la separación entre raíces y sufijos.

La descomposición de la palabra en [raiz + sufijos + desinencias] superó el estudio clásico de las declinaciones y facilitó el análisis comparativo de las estructuras morfológicas de las lenguas. Bopp tomó la noción de raíz presente en los hindúes cuyas formas base tenían un vocalismo mínimo; pero influenciado por el Romanticismo ${ }^{34}$, se propuso buscar el primer estado de la lengua y descubrir las raíces monosilábicas aisladas. Es en este aspecto, donde se puede notar la independencia de pensamiento de Bopp frente a los hindúes: "Las racines verbales ansi que les racines pronominales sont monosyllabiques ... Hormis tá régle qu monosylllabisme, les racines verbales ne sont soumisses a aucune aure condition restrictive; elles peuvent contenir un nombre très variable de lettres...-dans 'sta' 'se tenir', le 's' et le ' $t$ ' ont été réunis de toute antiquité, comme de prouvent toutes les langues indoeuropéenes; dans 'skand 'monter (tatin Scana-o), tâ double combinaison de deux consones au comprençementier ali a ginvaésta" raçine est un fait dont l'antuquité est prouvée par l'accord du sanskrit et du latin. D'un autre coté, une simple voyelle suffisai pour exprimer ane idéc verbale: c'est ce qu'atteste

33 Los gramáticos grecolatinos no distinguieron entre flexión y deivación. Un caso aislado fue el de Varrón, quien sí observó esta distinción al hablar de la 'declinatio voluntaria' y la 'declinatio naturalis'. El sistema gramatical de los 'modistas' también distinguió lo que más tarde en gramática se conocería con el nombre de formaciones por flexión y formaciones por derivación.

34 En realidad, Bopp fue ellingüista menos romántico de su época. En su obra, se aprecia un espíritu positivo, cuyo objetivo fue la comparación de las semejanzas de estructura entre las lenguas. En muchas ocasiones, Bopp no hizo caso de las observaciones de sus contemporáneos y adoptó una actitud independiente, lo que revela el fuerte sentido de su propio valor $\mathrm{y}$ originalidad. 
la racine signifiant 'aller', qui se retrouve dans presque tous les idiomes de la famille indo-européenne" ${ }^{35}$.

La noción de raíz fue estudiada con un sentido utópico y de modo metafórico, lo que impidió que Bopp obtuviera un análisis descriptivo de las unidades de la primera articulación como el que había logrado Panini. Bopp buscaba las raíces monosilábicas aisladas en las que se pudiera percibir la relación significante que va del sonido puro al sentido. Tomó el sánscrito como modelo puro original del desarrollo del lenguaje y sostuvo que sus raíces eran reveladoras de la estructura morfológica de otras lenguas, puesto que por comparación, permitían identificar nuevas raíces: “...le sanscrit se trouve, par sa structure, dans le rapport le plus intime avec le grec, le latin, les langues germaniques, etc. et que grace à la comparaison de cet idiome, on était enfin sur un terrain solide, non-seulement pour expliquer les relations qui unissent entre eux les deux idiomes appelés classiques, mais encore pour marquer les rapport qu'ils ont avec le germanique, le lithuanien, le slave"36.

Según Bopp, en la protolengua, los elementos semánticos primitivos se expresaban mediante morfemâs separados; de manera que la morfología de una palabra constituía una representación de los elementos de su significado. La expresión del mundo de ideâs habría sido paralela en su estructura, necesariamente, con la de la palabra expresada. Sus argumentos para la reconstrucción se basaban enanálisis semánticos que confirmaban estos puntos de vista cuantitativos.

\section{«Jorge Puccinelli Converso»}

Bopp mostró desinterés por los aspectos fonéticos y a diferencia de Panini, quien se interesó principalmente por la estructura de la lengua, a Bopp le atrajo más el tema de la relación entre forma y significado en el lenguaje. Kiparsky opina que ese afán de Bopp por encontrar la relación entre forma y significado constituyó la diferencia más importante entre Bopp y los neogramáticos, situación análoga a la que alguna vez existió entre Panini y otro representante de la escuela hindú, Katyayana.

Bopp terminó por reconocer que no es posible lograr una correspondencia exacta entre forma y significado, y que las reconstrucciones sólo produ-

35 BOPP, Franz; Grammaire Comparée des Langues Indo-européenes, trad. Michel Bréal, Paris, Imprimiere Impériale, 1846.

36 Ibid., p. 2. 
cían representaciones de las propiedades semánticas más importantes, nunca representaciones semánticas completas. Uno de los puntos más discutidos fue la derivación de la marca de tiempo mediante el prefijo negativo. Bopp explicó que la función de este prefijo consistía en negar el tiempo presente para indicar un tiempo pasado, pero no negaba el verbo. En esta interpretación, subyace el problema clásico sobre el alcance de la negación. Y es que el método comparativo de Bopp produjo una protomorfología que de alguna manera revelaba las relaciones lógicas.

Bopp distinguió dos clases de raíces: las pronominales, de las cuales se formaron las partículas, las conjunciones y las preposiciones; y las raíces no pronominales, concebidas como núcleos cerrados e inalterables que aparecen rodeados de otras sílabas indicadoras de conceptos gramaticales. "Il y à en sanskrit et dans les langues de la même famille deux classex de racines: la première classe, qui est de beaucoup la plus nombreuse, a producit des verbes et des noms (substantifs et adjectifs); car les noms ne dèrivent pas des verbes, ils se trouvent sup une même ligne vec eux et on même provenance. Nous apéllerons toutefois cette classe de racines, l'heure, ... racines verbales; ... De la seconde classe de racines dérivent des pronoms, toutes les prépositions primitives, des conjonctions et des particules; nous les nommons racines pronomoninales, laquelle est contenues, d'une façon plus ou moins cahéc, dans les prépositions, les conjonctions et les particules" ${ }^{37}$.

Bopp no adoptó el criterio de clasificación de Tas raíces de Panini, quien se había basado principalmente en el significado de los verbos, llegando a distinguir entre raíces verbales y no verbales. Bopp criticó, incluso, a los hindúes por haber creído que todas las palabras derivaban de las raíces verbales $^{38}$ : “...les grammariens indiens font venir tous les mots, y compris le pronoms, de racines verbales, quoique la plupart des themes pronominaux s'opposent, même sous le rapport de la forme, a une pareille derivation ... Il y à quelquefois identité fortuite entre une racine pronominale; par example entre $i$ 'aller' et $i$ 'celui ci'",39.

3) Ibid., t. 1 p. 221.

38 No se sabe con certeza si Panini estuvo de acuerdo con esta teoría de los hindúes.

$39 \quad$ Ibid, t. 1 p. 222. 
Entre las raíces verbales, ubicó el verbo abstracto, que unía un sujeto a un predicado, y los verbos atributivos, que añadían un atributo. Estos últimos fueron subdivididos en aquellos que sólo tienen flexión interna de la raíz, y aquellos que llevan inserto el verbo abstracto. Uno de los aspectos más importantes de la lingüística comparativa fue precisamente el estudio de la flexión y no propiamente de las raíces. En la obra Über das Konjugationssystem, referida a las flexiones verbales, el concepto de flexión sufre una transformación decisiva ${ }^{40}$. Bopp observó que las lenguas se asemejaban más en su modo de construcción que en las raíces, y señaló que el sánscrito retenía las flexiones originales mediante las cuales, podía recuperarse el sistema flexivo original de las lenguas comparadas. Como se sabe, el sánscrito era una lengua puramente flexiva, a diferencia del latin, las lenguas germánicas, el griego y el persa que habían sido afectadas por el método de afijación según un esquema lógico. El estudio de la flexión fue, pues, el punto de partida más importante para la reconstrucción histórica de las formas gramaticales. Durante mucho tiempo, las lenguas que se consideraban relacionadas coincidían en sus sistemas flexivos, de modo que esta coinciđencia se tomó como criterio para determinar la relación lingüística ${ }^{41}$.

En todas las lenguas que procedían del sánscrito, cualquier accidente se expresaba por medio de una flexión que era común a ellas y a la lengua originaria. El objetivo de la lingüística comparativa fue, entonces, buscar la similitud entre las lenguas en susjàspęctoscestructuraTes más ocultos. Bopp sostuvo la teoría de la aglutinaciǵn, según la cual las desinencias derivaban de raíces pronominales y de formas del verbo "ser" que se unían a las raíces verbales. En las formas verbales flexivas, podían hallarse los exponentes de la raíz (el atributo), la cópula y la persona (el sujeto). Bopp quería encontrar en todos los verbos, desinencias de la raíz "ser", el llamado verbo abstracto, verbo sustantivo o verbo originario. Así por ejemplo, la forma verbal 'potest' era analizada como 'pot-' (verbo atributo), 'es-' (cópula) y '- $t$ ' (el sujeto); y en el presente latino 'possum', aparecería el verbo abstracto 'esse' unido a una raíz atributiva.

La interpretación de las formas lingüísticas que hizo Bopp refleja su concepción de las lenguas como cuerpos naturales orgánicos que se forman

40 Mounin sugiere que Bopp y Schlegel habrian sido inventores de la noción de flexión. Véase G. Mounin, Historia de la Lingüistica, trad. Felisa Marcos, Madrid, Gredos, 1989, p. 180.

${ }^{4}$ Esto no implica que necesariamente, las lenguas con sistemas flexivos distintos no se relacionen, puede demostrarse relación entre lenguas que difieren en su sistema flexivo. 
según leyes determinadas y tienen un principio interno de vida. La estructura de la lengua se concibe, así, como un todo orgánico que se ramifica en todas sus significaciones por medio de flexiones o cambios internos. Bopp observó que las modificaciones gramaticales se expresaban tanto por la flexión de la raíz (cambio de la vocal interna y reduplicación), como por la adición de afijos. Los accidentes de los verbos, por ejemplo, se expresaban en sánscrito por medio de modificaciones de la raíz. Sin embargo, la noción de persona no se expresaba mediante un procedimiento de inflexión interna. Basándose en las semejanzas entre los pronombres y las marcas de persona, Bopp sostuvo que las terminaciones personales de los verbos habrían sido originariamente pronombres unidos a la raíz verbal: "Si la langue, dit-il, a employé, avec le génie prévoyant qui lui est propre, des signes simples pour représenter les idées simples des personnes, et sí nous voyons que les mémes nottions sont représentées de la même maniére dans les verbes et dans les pronoms, il s'ensuit que la lettre avatt a l'origine une signification et qu'elle y est restée fidele. S'il y a eu autrefois une raison pour que mam signifiat 'moi' et pour que tam signifiat 'lui', c'est sans aucune doute la même raison qui fait que bhava-mi signifie 'je suis' et que bhava-ti sugnifie 'il est'. Du moment que la langue marquait les personnes dans le verbe en joignant extérieurement des letres a la racine, elle n'en ouvait légitemement choisir d'autres que celles qui, depuis l'origine du langage, représentaient l'idée de ces personnes" ${ }^{42}$.

Al observar las diversas variaciones significativas que no se expresaban con la modificación de la raiz, Bopp abandonó una definición muy restringida de flexión e incluyó ến estéprocesonel cambio/aełašs terminaciones. En una interpretación como la de Schlegel, sólo se habría reconocido como flexión en las lenguas indoeuropeas a la reduplicación. Bopp, sin embargo, sostuvo que el origen de la flexión en indoeuropeo habría sido la adjunción de sílabas exteriores a la raíz, y de este modo, añadió al concepto de flexión, la adición de afijos: 'Par flexión, Fr: de Schlegel entend le changement interne du son radical, ou la modification interne de la racine qu'il se oppose a l'adjonction externe d'une syllabe. Mais quand en grec de ou de se forment qu'este-ce que les formes sinon des complements externes qui viennent s'ajouter à une racine invariable ou changeant seulement la quantité de la voyelle? Si l'on entend done par flexion une modification interne de la racine, le sanscrit, le grec, etc. n'auront guere d'autre flexion que redoublement, qui est formé a l'aide

42 BOPP, Franz; Systeme de conjugaison de la langue sanscrite, p. 147, cit. por Michel Bréal en F. Bopp, Grammaire Comparée des langues indoeuropéennes, p. xxv. 
des ressources de la racine même ... car si la racine a la faculté d'exprimer les idées de rapport par de changement internes, comment en peut-on conclure pur cette même racine (qui reste invariable a l'interieur) la faculté de se developper indéfiniment a l'aide de syllabes étrangeres s'ajoutant du dehors ... Mais quand même il serait impossible de reconnnaitre avec certitude l'origine d'une seule de ces flexion, il n'en serait pas moins certain our cela que l'adjonction de syllabes extérieures ast le véritable principe de la grammaire indoeuropéene ${ }^{43}$.

Lejos de postular que la flexión sólo implica adición de afijos, Bopp conservó la definición de flexión como variación interna de la raíz y analizó la inserción del verbo abstracto como un caso de composición. Con este análisis, Bopp logró describir la flexión en todos sus aspectos y reconoció muy bien las raíces, tal como lo habían hecho los hindúes, incluido Panini. Puede relacionarse esta precisión de Bopp con las operaciones indicadas por las reglas paninianas: adición y sustitución. El conocimiento de la descripción de Panini le ayudó a Bopp a reconocer como flexión tanto a la sustitución (modificación interna del radical), como a la adición de formas lingüísticas mínimas.

Finalmente, cabe señalar que Bopp no hizo referencia a la sintaxis y es pơsible que también en ello haya influido la obra de Panini, que aunque sí contiene un análisis de las relaciones entre palabras, su interpretación requiere un estudio profundo que los comparatistas no lograron, debido quizás, a su afán por reconstruir las formas-6figinales delás-lenguas.

\section{CONCLUSIÓN}

Mediante el análisis de las nociones de "raíz", "flexión" y "derivación" en la obra de Panini y en la obra clásica de la gramática comparativa, me ha interesado mostrar la influencia de Panini en la obra de Bopp, particularmente en el reconocimiento de la noción de raíz, en la distinción entre la raíz y el tema, así como en la precisión del concepto de flexión. Las divergencias que en algún momento he señalado podrían explicarse, tal vez, si se tuvieran en cuenta los distintos fines que ambos gramáticos se propusieron.

${ }^{43}$ BOPP, F.; Grammaire comparée des langues indo-européennes, t. 1, pp. 226-227. 


\title{
BIBLIOGRAFÍA
}

\begin{abstract}
ALLEN, William Sidney
1953

Phonetics in Ancient India, London, Oxford University Press.

1955

"Zero and Panini", Indian Linguistics, 16, pp. 106-113.
\end{abstract}

ARENS, Hans

1975

La Lingüística, trad. José María Díaz Regañón López, Madrid, Gredos, 2 t.

BAHULIKAR, Saudammi

1973

"Concerning the Structure of Panini's Astadhyayi", Indian Linguistics, 34 2, pp. 75-99.

BARLINGAY, S.S.

1965

A modern introduction to Indian Logic, N. Delhi, National Publishing House.

BENVENISTE, Emile

1971

Problemas de lingüistica general, trad. Juan Almela, Méxi$\mathrm{co}$, Siglo XXI.

BLOOMFIELD, Leonard

1964

Lenguaje, trad. Alma Flor Ada de Zubizarreta, Lima, Universidad Nacional Mayor de San Marcos.

1929

sobre Bruno Libich, Kondornanz Panini-Candra, Breslau, Verlag von M.\&H., Marcus, Language, 5, pp. 267-276.

BOPP, Franz

1846

Grammaire Comparée des Langues Indo-européennes, trad. Michel Bréal, Paris, Imprimerie Impériale, 5 ts.

CARDONA, Georg

1976

Panini: a survey of research, The Hague, Mouton \& Co.

1988

Panini, his work and its traditions, N. Delhi, Motilal Banasidass. 
DIDERISCHE, Paul

1974

"The Foundation of Comparative Linguistics: Revolution or Continuation?", en Studies in the History of Liguistics. Traditions and Paradigms, ed. Dell Hymes, Bloomingon, Indiana University Press, pp. 277-306.

KIPARSKY

"From Paleogrammarians to Neogrammarians", en Studies in the History of Linguistics Traditions and Paradigms, pp. 331-345.

1979

Panini as a Variationist, Cambridge, Massachussets Institute of Technology.

MARTINEAU, Roussell

1966

"Franz Bopp", en Portraits of Linguistics. A Biographical Source Book for the History of Western Linguistics, ed. Thomas A. Sebeok. Bloomington, Indiana University Press, pp. 200-206.

MATTHEWS, P.H.

1974

Morphology, An introduction to the theory of word structure, New York, Cambridge University Press.

MISRA, Vidya

1966

\section{Biblioteca de Letras}

The deseriptivetechinque of Pdmint, The Hague, Mouton.

PANINI

1987

Astadhyayi of Panini, trad. Sumítra Mangesh Katre, Austin, University of Texas Press.

1991

Paninian Studies, ed. Madhav Desphande, Michigan, University of Michigan.

PEDERSEN, Holger

1983

A Glance at the History of Linguistics with particular regard to the historical study of phonology, trad. Caroline C. Henriksen, ed. Konrad Koerner, Amsterdam, John Benjamins Publishing Company. 
PIMBORG, Jan

1975

"Classical Antiquity", Current Trends in Linguistics, pp. 69126.

RENOU, Louis

1969

"Panini”, Current Trends in Linguistics, 5, pp. 481-498.

ROBINS, R.H.

1987

Breve Historia de la lingüística, trad. Enrique Alcaraz Varo, Madrid, Paraninfo.

ROCHES, Rossane

1969

"The concept of verbal root in Indian grammar: à propos of Panini 1.3.1.", Foundations of Language, 5, pp. 73-82.

1975 "India", Current Trends in Linguistics, 13, pp. 3-67.

ROUSSEAU, Jean 1980

"Flexion et racine: Trois étapes de leur constitution: J.C. Adelung, F. Schlegel, F. Bopp", en Progress in Linguistics Historiography, ed. Konrad Koerner, Amsterdam, John Benjamins North America, pp. 235-248.

SCALISE, Sergio 1987

Morfölogíagenerativa trad. Iosé Pazó, Madrid, Alianza Universidactuccinelli Converso»

SHEFTS, Betty 1961

Grammatical Method in Panini, His treatment of Sanskrit Present Stems, New Haven Connecticut, American Oriental Society.

SINGH, Rajendra \& FORD, Alan

"Flexion, derivation et Panini", en Progress in Linguistics, Historiography, pp. 323-332.

STAAL, J.F.

"The Origin and Development of Linguistics in India", en Studies in the History of Linguistics Traditions and Paradigms, pp. 63-74. 
VAN NOOTEN, B.A.

1967

"Panini's replacement technique and the active finite verb", Language, 43, pp. 883-894.

VIERBUNG, Peter

"The Background to the Linguistics Conceptions of Franz Bopp", en Portraits of Linguistics. A Biographical Source Book for the History of Western Linguistics, pp. 221-250.

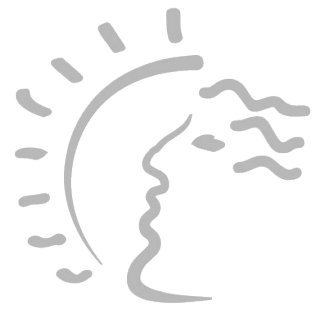

Biblioteca de Letras «Jorge Puccinelli Converso» 